\title{
ARTICLE
}

\section{Comparison of the Fukushima radioactive mapping by two different aerial radiation monitoring systems}

\author{
Byoung-Jik Kim ${ }^{a^{*}}$, Miyuki Sasaki ${ }^{\mathrm{b}}$ and Yukihisa Sanada ${ }^{\mathrm{b}}$ \\ ${ }^{a}$ Korea Institute of Nuclear Safety, 62 Gwahak-ro, Yuseong-gu, Daejeon 34142, Republic of Korea; ${ }^{b}$ Fukushima \\ Environmental Safety Center, Japan Atomic Energy Agency, 45-169 Sukakeba, Kaibama-aza, Haramachi, Minamisoma, \\ Fukushima, 975-0036, Japan
}

\begin{abstract}
A radiation monitoring system using unmanned aerial vehicles (UAVs) was developed for post-nuclear accidents by the Korea Institute of Nuclear Safety (KINS). The Japan Atomic Energy Agency (JAEA) has been conducted radiation monitoring in the Fukushima area and undertaking research and development of related technology. Considering future large-scale disasters, it is important that measurement methods using UAVs are unified between the neighbor countries. Two organizations in neighbor countries attempted technical collaborations to compare results obtained from different methods. In 2015, measurements were carried out in an area within $10 \mathrm{~km}$ from the Fukushima Dai-ichi Nuclear Power Station (FDNPS), where an accident occurred due to the Great East Japan Earthquake and where contamination remains and access is controlled. Corrections to convert the coefficients from detectors in air to the radiation dose or radioactivity concentration on the surface were carried out in areas previously surveyed as flat and relatively evenly contaminated. Explorations of contamination mapping were conducted in river basins, with contaminants appearing in different water and soil contours, which were expected to make intuitive comparisons easier for multiple mappings. We used a Japanese unmanned helicopter, which is used for agricultural applications such as the spraying of pesticides. The measurement system of JAEA and KINS was installed and the same route was flown once in each case.
\end{abstract}

Keywords: aerial radiation monitoring; radioactive map; unmanned aerial vehicle; accident of Fukushima Dai-ichi Nuclear Power Station; Nuclear Emergency Preparedness

\section{Introduction}

In the event of an accident involving a release of radioactive contamination, it is necessary to measure the pollution within a short period of time and map the result on a geographic map to reassure residents when carrying out measures to protect them [1]. Rapid radiometric measurements over a large area (with a radius of at least a few kilometers) can be challenging $[2,3]$. Aerial measurements using aircraft are the most effective and efficient means thus far. The accident of Fukushima Dai-ichi Nuclear Power Station (FDNPS) and the response underlined the importance of aerial gamma survey technology, which enabled emergency decision makers to be aware of the state of radioactive contamination [4,5]. This technique, which has been widely used in relation to the FDNPS accident, mainly focuses on calibration to convert counts in the air to the ground radiation dose or radioactivity concentration and mapping to project the dose or the concentration back to the ground.

*Corresponding author. Email: bjkim@kins.re.kr
This type of mapping refers to the drawing of radioactive contamination maps by integrating the functions of a metrology system (e.g., radiation counts, location or altitude) and a calibration process, and it can be divided into several subdivision processes, as follows. 1) Measurement by system (radiation coefficient, position, altitude). 2) Correction methods such as excluding radiation not coming from the surface. 3) Calibration with radiation attenuation according to the altitude. 4) Corresponding measurement results after calibration to a radiation value (e.g., radioactivity concentration) according to the ground position.

There is a considerable degree of uncertainty related to the task of mapping ground surface locations to radiation values. The process of estimating or limiting the uncertainty is also complicated. In order to use this data as information related to protective measures in the event of a pollution accident, it is necessary to secure its reliability. It is not easy to demonstrate validity in these cases with a single mapping (metrology system and calibration), and it is dangerous to trust the outcomes without such a procedure.

It is a relatively secure method when operating 
multiple systems under identical conditions, comparing the results, and recognizing a certain level of validity when they are similar, although validation in such a case remains incomplete. In the present study, researchers from two countries compared radioactive maps from each respective system.

\section{Materials and methods}

\subsection{Measurement date and area}

On November 3 and 4, 2015, measurements were carried out in the contaminated area around the FDNPS. Measurements were carried out in an area within $10 \mathrm{~km}$ from FDNPS, where an accident occurred due to the March 2011 earthquake in eastern Japan, and where the contamination remains and the access is controlled (Figure 1 (a)). Explorations for contamination mapping were conducted in river basins, with contaminants appearing in different water and soil contours, which were expected to make intuitive comparisons easier for the mappings (Figure 1 (b)). Calibrations to convert the coefficients from the detector in air to the radiation dose or radioactivity concentration on the surface were carried out in areas previously surveyed as flat and evenly contaminated (Figure 1 (c)).

\subsection{Unmanned aerial vehicle}

The autonomous unmanned helicopter (RMAX G1, Yamaha Motor Co. Ltd., Shizuoka, Japan) owned by the Japan Atomic Energy Agency (JAEA) was used. With a total length of $3,630 \mathrm{~mm}$, a total width of $720 \mathrm{~mm}$ and a total height of $1,220 \mathrm{~mm}$, this unmanned helicopter has a top speed of $72 \mathrm{~km} \mathrm{~h}^{-1}$ and a maximum weight of $10 \mathrm{~kg}$ onboard. The measurement systems of JAEA and the Korea Institute of Nuclear Safety (KINS) were installed and the same route was flown once in each case.

\subsection{Flight path}

The RMAX flew through the designed route under the control of a ground station. The RMAX followed the previously designed flight plan (Figure 2). The altitude was set at 30 meters, the flight interval was 30 meters, and the flight speed was $5 \mathrm{~km} \mathrm{~h}^{-1}$ in the plan.

For detector calibration, the gamma spectra were measured at heights of $10,20,30,40,50,70$ and 100

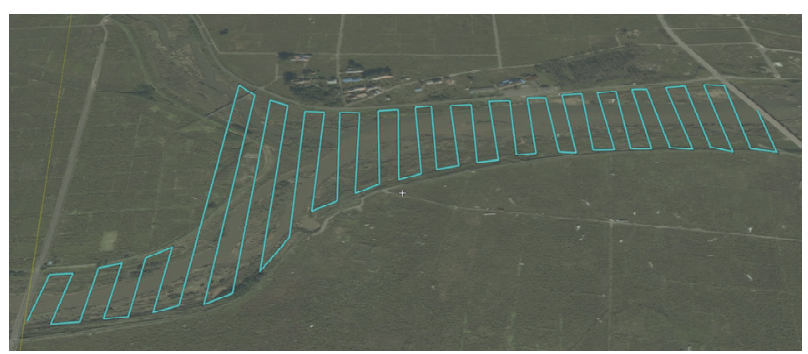

Figure 2. The aircraft flew along a prescribed route.

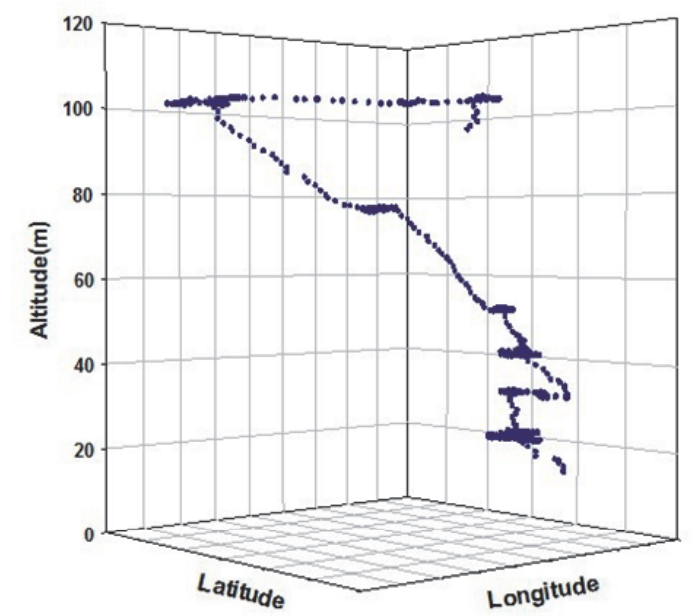

Figure 3. Attenuation according to the altitude was calibrated.
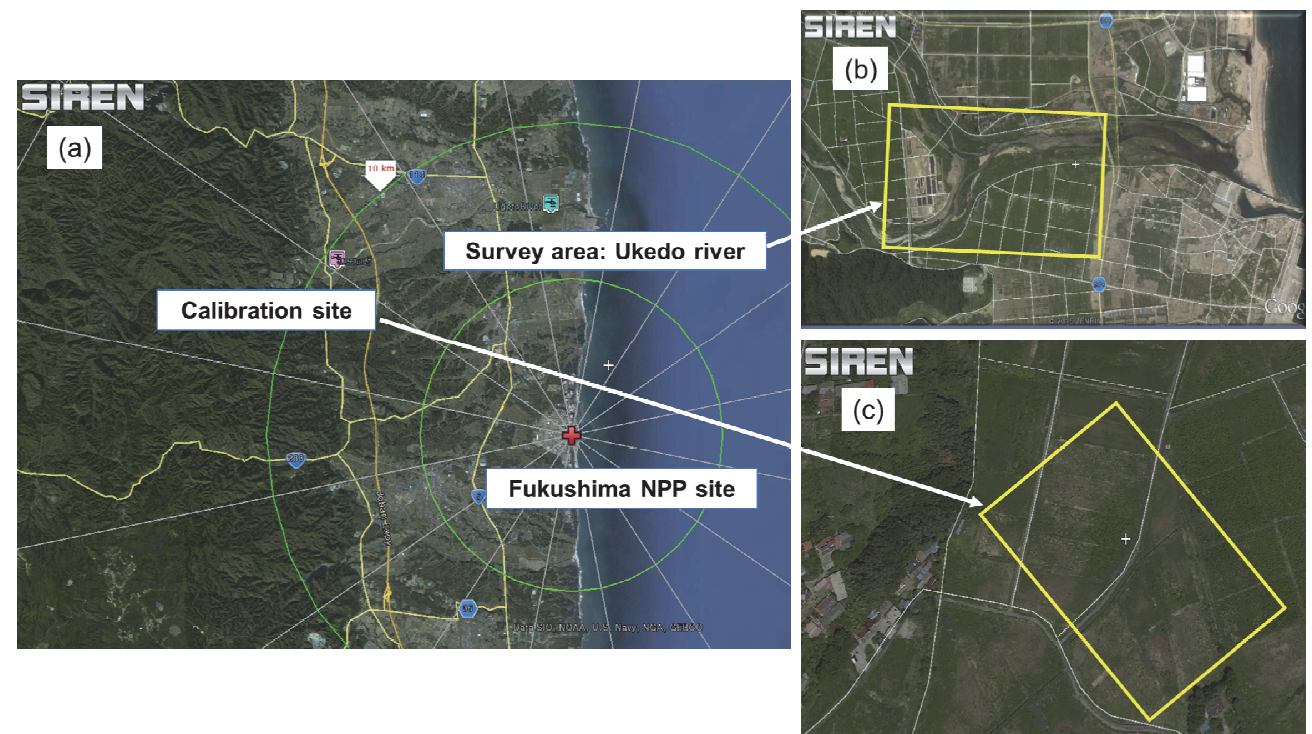

Figure 1. (a) Measurements were performed in an area within $10 \mathrm{~km}$ from FDNPS. (b) Survey flights were conducted in river basins. (c) The calibration site was located in a flat area. 
meters. Figure 3 shows the movement of the RMAX for calibration.

\subsection{Radiation measurement system}

Specifications of radiation measurement system of JAEA and KINS are shown by Table 1. Both systems were used $\mathrm{LaBr}_{3}(\mathrm{Ce})$ scintillators. Scintillator size of KINS is bigger than JAEA's. The data from some detectors were sent to the ground station via a radio channel independent of the helicopter control signal channels. The helicopter's position and detectors' total count rate information was displayed on a map of the ground station in real time. A configuration example of this system and the detectors implemented with the helicopter are shown in Figure 4. Specifically, KINS instrumentation was controlled via a wireless internet connection and was monitored at the headquarters of KINS, located in Daejeon, Korea.

For calibration of aerial measurement system, ground measurements were taken using a handheld $\mathrm{NaI}$ scintillation survey-meter (TCS-171B, Hitachi Co. Ltd., Tokyo, Japan), to record ambient gamma dose rate at 1 $\mathrm{m}$ above the ground level (agl.).

Table 1. Specifications of KINS and JAEA system.

\begin{tabular}{lcc} 
& KINS & JAEA \\
\hline $\begin{array}{l}\text { Detector } \\
\text { Dimension }\end{array}$ & $\mathrm{LaBr}_{3}(\mathrm{Ce})$ & $\mathrm{LaBr}_{3}(\mathrm{Ce})$ \\
Volume (L) & 0.21 & $1.5^{\prime \prime} \phi \times 1.5^{\prime \prime} \mathrm{H} \times 3$ ea \\
$\begin{array}{l}\text { Attenuation factor } \\
\left(\mathrm{AF}: \mathrm{m}^{-1}\right)\end{array}$ & 0.0078 & 0.13 \\
$\begin{array}{l}\text { Conversion factor } \\
\text { for dose rate } \\
\left(\mathrm{CD}: \mathrm{cps} \mu \mathrm{Sv}^{-1} \mathrm{~h}\right)\end{array}$ & & 0.0061 \\
\hline
\end{tabular}

\subsection{Data acquisition and analysis}

KINS collected data through real-time communications during the flight, and JAEA collected data through a wired connection after the flight. The raw data on both sides was exchanged. Figure 5 shows KINS' procedure used to create a radioactivity map via the survey and the calibration steps. It's very common,

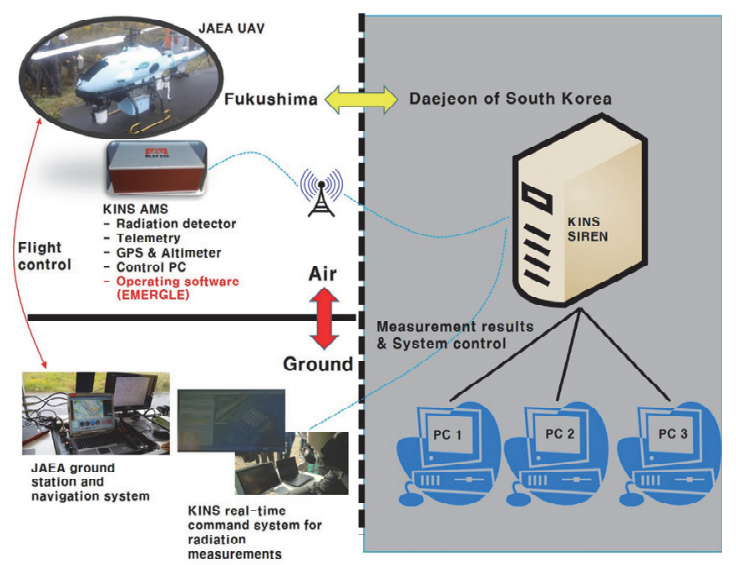

Figure 4. KINS instrumentation was controlled and monitored via a wireless internet connection.

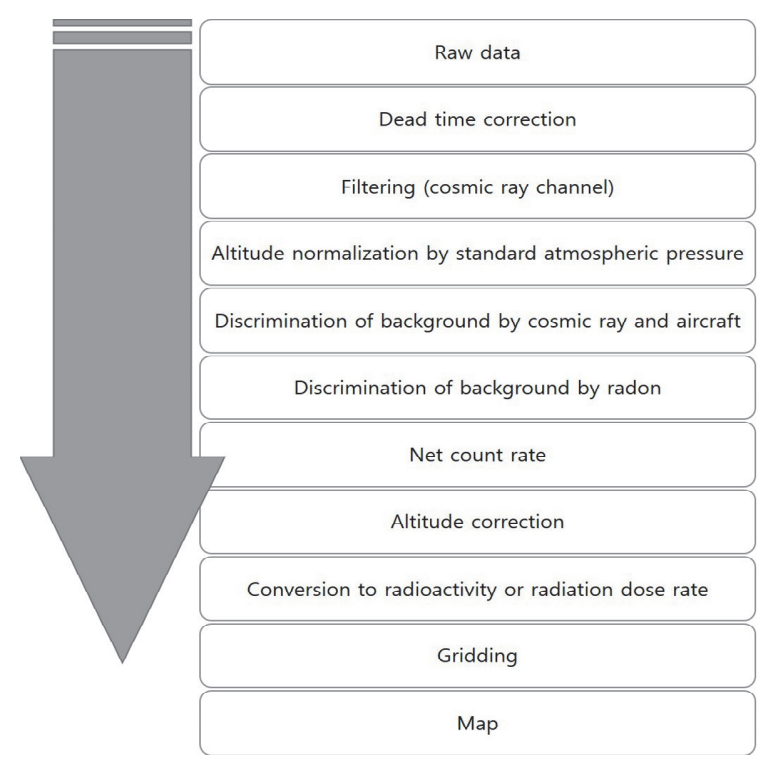

Figure 5. KINS' procedure consists of several steps, from raw data procuring to the drawing of a map.

and the JAEA procedure is conceptually similar to that.

\section{Results and discussion}

\subsection{Calibration}

In calibration site, the mean dose rate of the ground survey was $4.4 \pm 0.7 \mu \mathrm{Sv} \mathrm{h}{ }^{-1}$ for KINS and $5.5 \pm 0.6 \mu \mathrm{Sv}$ $\mathrm{h}^{-1}$ for JAEA. The difference of approximately $20 \%$ is presumably due to the different instruments used, which were not calibrated to the same standard.

The curve of the altitude vs. the count rate was plotted and the linear attenuation factor (AF) was obtained. The difference of about $15 \%$ between the two results may be due to the use of detectors with different volumes and geometries, and it is necessary to check this later with modeling tools.

From the mean dose rate of the ground survey and the linear attenuation coefficient, the radiation doses to the count rate conversion factor to dose rate (CD) $1 \mathrm{~m}$ agl. were calculated as 5,900 and 4,100 (cps $\left.\mu \mathrm{Sv}^{-1} \mathrm{~h}\right)$, respectively. There was a difference of about $30 \%$. This is interpreted as a result of combining the ground survey results with a difference of about $20 \%$ and the difference in the line attenuation coefficients of $15 \%$.

\subsection{Mapping}

Both groups' raw data and CD were exchanged. Each group draws one map with its own data and $\mathrm{CD}$, and another map with the opponent's data and CD. The four maps (Figure 6) intuitively show similarities with nearly identical locations of the hottest spot. There is a need for a method capable of assessing how similar these outcomes are. Image pattern comparison methods are considered as candidates. 


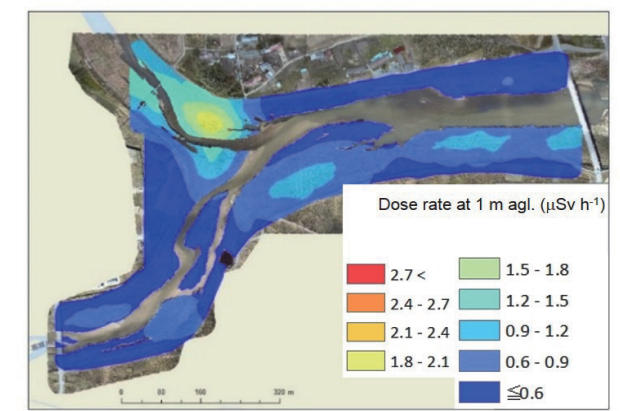

(a) JAEA data: analyzed by JAEA

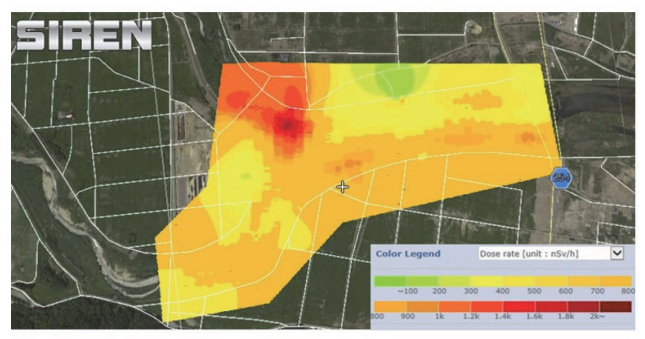

(c) KINS data: analyzed by KINS

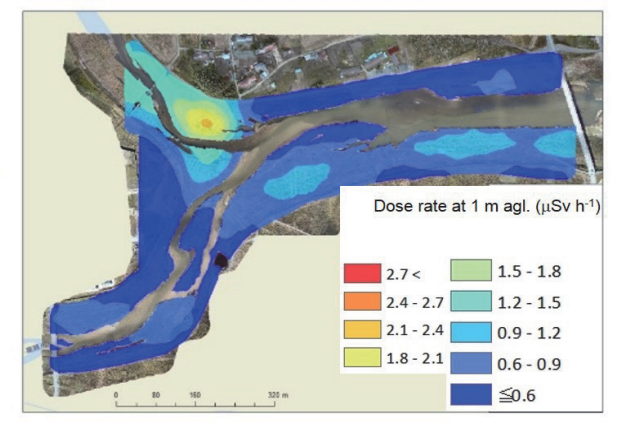

(b) KINS data: analyzed by JAEA

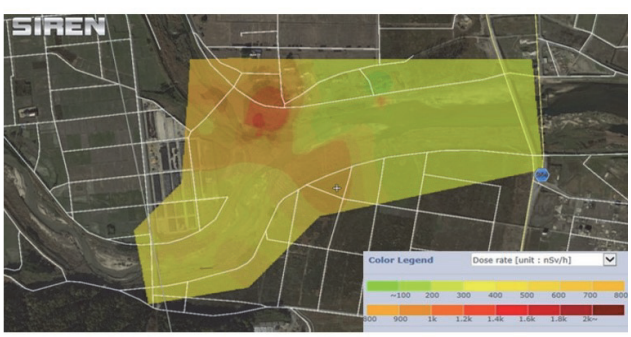

(d) JAEA data: analyzed by KINS

Figure 6. Contour map of dose rate. (a) and (b) JAEA data and KINS data were analyzed by JAEA method. (c) and (d) KINS data and JAEA data were analyzed by KINS method.

\subsection{Ambient gamma dose rate}

Two corresponding ambient gamma dose rates on the two maps drawn by JAEA (Figures 6 (a) and (b)) were plotted at each point (Figure 7). A strong correlation was confirmed. Both instruments can be considered as capable of providing similar raw data for mapping.

The maps drawn by KINS and JAEA were set to a grid in differ ways; hence, a comparison was not plotted.

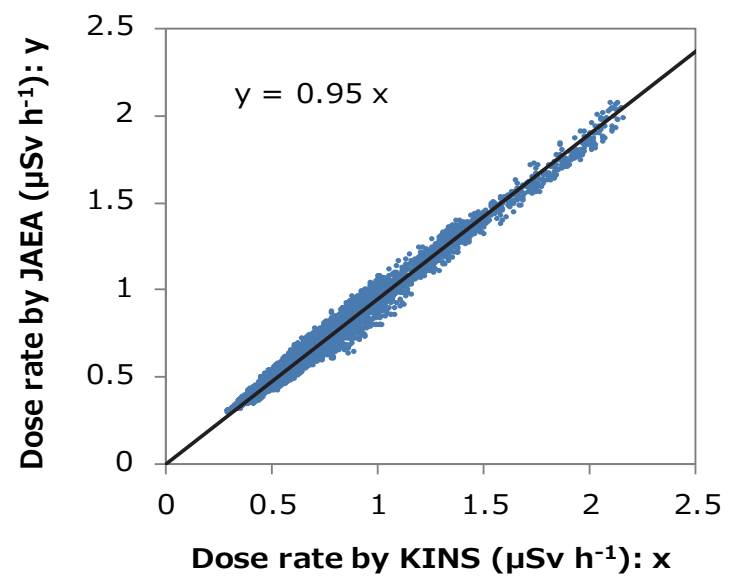

Figure 7. The ambient gamma dose rates by the two parties are plotted.

\section{Conclusion}

Ground surface contamination mapping by aerial radiation measurements is a strategy that must be prepared for immediate operation when radioactive contamination occurs. However, before an incident occurs, it is difficult to effectively verify the validity of such an operation without a polluted environment.
Cross-checking with proven pollution equipment is considered as a useful preparation step before the operation of new equipment.

\section{Acknowledgement}

We thank Mr. Y. Nishizawa, Mr. M. Imura, Mr. Y. Takamura in OYO and Mr. G. P. Lee in RADSEARCH Co. Ltd. for their cooperation in taking measurements and processing data. We are also grateful to Dr. T. Torii, Dr. S. Nakayama in JAEA, Mr. H. Y. Choi and Dr. W. J. Kim in KINS for giving many valuable comments.

\section{References}

[1] M. Takeishi, M. Shibamichi, A. Malins, H. Kurikami, M. Murakami, J. Saegusa and M. Yoneya, Using two detectors concurrently to monitor ambient dose equivalent rates in vehicle surveys of radiocesium contaminated land, J. Env. Rad. 177 (2017), pp. 1-12.

[2] R. Kleinschmidt and D. Watson, Terrestrial gamma radiation baseline mapping using ultra low density sampling methods, J. Env. Rad. 151 (2016), pp. 609-622.

[3] K. Saito and Y. Onda, Outline of the national mapping projects implemented after the Fukushima accident, J. Env. Rad. 139 (2015), pp. 240-249.

[4] Y. Sanada and T. Torii, Aerial radiation monitoring around the Fukushima Dai-ichi nuclear power plant using an unmanned helicopter, J. Env. Rad. 139 (2015), pp. 294-299.

[5] Y. Sanada, T. Orita and T. Torii, Temporal variation of dose rate distribution around the Fukushima Daiichi nuclear power station using unmanned helicopter, Appl. Rad. and Isot. 118 (2016), pp. 308-316. 